\title{
Efficacy and safety of fremanezumab in clinical trial participants aged $\geq 60$ years with episodic or chronic migraine: pooled results from 3 randomized, double-blind, placebo-controlled phase 3 studies
}

Stephanie J. Nahas ${ }^{*}$, Steffen Naegel ${ }^{2}$, Joshua M. Cohen ${ }^{3}$, Xiaoping Ning ${ }^{3}$, Lindsay Janka ${ }^{3}$, Verena Ramirez Campos ${ }^{3}$, Lynda J. Krasenbaum³ ${ }^{3}$ Dagny Holle-Lee ${ }^{4}$, David Kudrow ${ }^{5}$ and Christian Lampl ${ }^{6,7}$

\begin{abstract}
Background: Although migraine is less common in older people, preventive treatment of migraine in these individuals may be more challenging due to the presence of multiple comorbidities and polypharmacy. Additionally, evidence for migraine treatment efficacy, safety, and tolerability is limited in this population. We evaluated efficacy, safety, and tolerability of fremanezumab, a fully humanized monoclonal antibody (IgG2 $\Delta a)$ that selectively targets calcitonin gene-related peptide (CGRP), in clinical trial participants aged $\geq 60$ years with episodic migraine (EM) or chronic migraine (CM).

Methods: This analysis included data from 3 randomized, double-blind, placebo-controlled phase 3 studies: the HALO EM study, HALO CM study, and FOCUS study in participants with EM or CM and prior inadequate response to 2-4 migraine preventive medication classes. Participants in all studies were randomized 1:1:1 to receive 12 weeks of subcutaneous treatment with quarterly fremanezumab (Months 1/2/3: EM/CM, $675 \mathrm{mg} /$ placebo/placebo), monthly fremanezumab (Months 1/2/3: EM, 225 mg/225 mg/225 mg; CM, 675 mg/225 mg/225 mg), or matched monthly placebo.

Results: These pooled analyses included 246 participants aged $\geq 60$ years. Reductions in monthly migraine days from baseline over 12 weeks were significantly greater with fremanezumab (least-squares mean change from baseline [standard error]: quarterly fremanezumab, -4.3 [0.59]; monthly fremanezumab, -4.6 [0.54]) versus placebo (placebo, -2.3 [0.57]; both $P<0.01$ vs placebo). As early as Week 1, significant reductions from baseline in weekly migraine days were observed with fremanezumab versus placebo (both $P<0.01$ ). With fremanezumab treatment versus placebo, a significantly higher proportion of participants achieved $\geq 50 \%$ reduction in monthly migraine days, and significant improvements in disability and quality-of-life outcomes were observed $(P<0.05)$. Proportions of participants experiencing serious adverse events and adverse events leading to discontinuation were low and
\end{abstract}

\footnotetext{
* Correspondence: Stephanie.Nahas@jefferson.edu

${ }^{1}$ Department of Neurology, Thomas Jefferson University, Philadelphia, PA, USA

Full list of author information is available at the end of the article
}

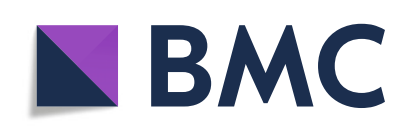

( ) The Author(s). 2021, corrected publication 2022. Open Access This article is licensed under a Creative Commons Attribution 4.0 International License, which permits use, sharing, adaptation, distribution and reproduction in any medium or format, as long as you give appropriate credit to the original author(s) and the source, provide a link to the Creative Commons licence, and indicate if changes were made. The images or other third party material in this article are included in the article's Creative Commons licence, unless indicated otherwise in a credit line to the material. If material is not included in the article's Creative Commons licence and your intended use is not permitted by statutory regulation or exceeds the permitted use, you will need to obtain permission directly from the copyright holder. To view a copy of this licence, visit http://creativecommons.org/ licenses/by/4.0/. The Creative Commons Public Domain Dedication waiver (http://creativecommons.org/publicdomain/zero/1. 0/) applies to the data made available in this article, unless otherwise stated in a credit line to the data. 
similar in the fremanezumab and placebo groups. Efficacy and safety results were comparable to the overall pooled population $(N=2843)$.

Conclusions: This pooled subgroup analysis demonstrates that fremanezumab treatment is efficacious and welltolerated over 12 weeks in participants aged $\geq 60$ years with EM or CM. These data may help healthcare providers with clinical decision making and preventive treatment selection for older patients with migraine.

Trial registration: ClinicalTrials.gov identifiers: HALO CM: NCT02621931; HALO EM: NCT02629861; FOCUS: NCT03308968.

Keywords: Episodic migraine, Chronic migraine, Fremanezumab, CGRP, Older age

\section{Background}

Migraine is the second leading cause of years lived with disability globally and is associated with a substantial negative impact on health-related quality of life [1-4]. Although migraine is less common in older people, a high prevalence of psychiatric disorders, such as depression, anxiety, and sleep disturbances, and the presence of multiple comorbidities, such as cardiovascular (CV) disorders and diabetes, may be associated with even further worsening in quality of life [5-9]. For example, individuals with migraine may have a more than 5 times greater risk of developing major depressive disorder compared with those without migraine [10-13], and that increased risk of depression is also observed in older people with migraine [8]. In addition, preventive treatment of migraine in older patients may be more challenging due to polypharmacy, especially with respect to medications used for comorbidities, and concerns around cognitive impairment, influenced by comorbidities, medications, and lifestyle [5, 7].

Treatment for patients with migraine includes both acute and preventive medications. For years, preventive treatment options have included anticonvulsants, antidepressants, antihypertensives (eg, $\beta$-blockers), flunarizine, and onabotulinumtoxinA $[14,15]$. However, these preventive treatments are not specific to migraine and are often unsatisfactory due to lack of efficacy, intolerability, and poor adherence [16-18]. Some of these may also have contraindications, especially in older patients.

There are currently 4 monoclonal antibodies (mAbs) targeting the calcitonin gene-related peptide (CGRP) pathway that are approved by the US Food and Drug Administration (FDA) for the preventive treatment of migraine [19-22]. Fremanezumab, a fully humanized $\mathrm{mAb}(\operatorname{IgG} 2 \Delta \mathrm{a})$ that selectively targets CGRP, has proven efficacy for the preventive treatment of migraine in adults [23-25]. Three randomized, double-blind, placebo-controlled, phase 3 trials have demonstrated that fremanezumab is well tolerated and efficacious in the preventive treatment of episodic migraine (EM) and chronic migraine (CM), even in individuals with difficult-to-treat migraine [23-25]. Long-term safety and efficacy of fremanezumab treatment was also demonstrated for up to 12 months in parallel-group phase 3 studies in participants with EM or CM [26].

The analyses presented here aim to evaluate the efficacy, safety, and tolerability of fremanezumab in participants $\geq 60$ years of age with EM or CM, which would add to the presently limited body of evidence regarding migraine treatment efficacy, safety, and tolerability in this population [5]. Given the worldwide increase in life expectancy, migraine in older age is likely to become an increasing issue over the next 40 years, with management likely confounded by other health problems and consequent association with polypharmacy. Therefore, these analyses with a selected age cutoff of $\geq 60$ years were performed.

\section{Methods}

\section{Study design}

This was a pooled subgroup analysis including data from 3 international, multicenter, randomized, double-blind, placebo-controlled, parallel-group, phase 3 trials in participants with CM and EM: HALO CM (ClinicalTrials.gov Identifier: NCT02621931), which included participants with CM; HALO EM (ClinicalTrials.gov Identifier: NCT02629861), which included participants with EM; and FOCUS (ClinicalTrials.gov Identifier: NCT03308968), which included participants with CM or EM who had a documented inadequate response or contraindication to 2 to 4 classes of prior migraine preventive medications. Detailed methods and study designs for the HALO and FOCUS studies have been previously reported [23-25] and are briefly summarized here.

\section{Participant population \\ HALO CM and HALO EM}

Participants eligible for the HALO studies included adults (18-70 years of age) with a history of migraine per International Classification of Headache Disorders (ICHD)- 3 beta criteria for $\geq 12$ months prior to screening with onset at $\leq 50$ years of age. In the HALO CM study, 
$\mathrm{CM}$ was defined as headache on $\geq 15$ days per month, with $\geq 8$ days fulfilling ICHD-3 beta criteria for migraine, probable migraine, or use of triptan or ergot medications, over a period of 3 months [25]. In the HALO EM study, EM was defined as headache on 6 to 14 days per month, with $\geq 4$ days fulfilling ICHD-3 beta criteria for migraine, probable migraine, or use of triptan or ergot medications [23]. Participants were excluded in both trials for use of onabotulinumtoxinA in the 4 months before screening, use of opioids or barbiturates on $\geq 4$ days per month, use of interventions or devices for migraine in the 2 months before screening, or previous failure from $\geq 2$ medication clusters after $\geq 3$ months of treatment (antiepileptics, calcium channel blockers, antidepressants, beta blockers) [23, 25]. Up to $30 \%$ of participants were permitted the use of 1 preventive migraine medication if the dose was stable from $\geq 2$ months before the pretreatment period to the end of the treatment period $[23,25]$.

\section{FOCUS}

Eligible participants for the FOCUS study included the same adult population as the HALO studies but with 1 key difference: eligible participants also had documented inadequate response or contraindication within the past 10 years to 2 to 4 of the following classes of prior migraine preventive medications: $\beta$-blockers, anticonvulsants, tricyclic antidepressants, calcium channel blockers, angiotensin II receptor antagonists, onabotulinumtoxinA, and valproic acid [24]. An inadequate response was defined as documentation in their medical record of no clinically meaningful improvement (per the treating physician's judgment) after 3 months of stably dosed treatment or discontinuation due to poor tolerability [24].

\section{Standard protocol approvals, registrations, and participant consents}

All 3 studies were conducted in accordance with their respective study protocols and the International Conference for Harmonisation guidelines for Good Clinical Practice, the Declaration of Helsinki, and relevant national and local regulations. The study protocols were approved by the appropriate ethics committees and institutional review boards. Participants provided written informed consent prior to performing any study procedure or assessment [23-25].

\section{Study design}

All 3 studies included a screening visit, a 28-day pretreatment period, a 12-week treatment period (doubleblind and placebo-controlled), and a final evaluation at Week 12. Enrolled participants were randomly assigned 1:1:1 to receive subcutaneous quarterly fremanezumab
(Month 1/2/3: $675 \mathrm{mg} /$ placebo/placebo), monthly fremanezumab (Month 1/2/3: CM, $675 \mathrm{mg} / 225 \mathrm{mg} / 225 \mathrm{mg}$; EM, $225 \mathrm{mg} / 225 \mathrm{mg} / 225 \mathrm{mg}$ ), or matched monthly placebo. Efficacy was evaluated using information entered by participants in a daily electronic headache diary throughout the treatment period [23-25].

\section{Outcome measures}

Post hoc analyses were conducted using these pooled data to assess the efficacy and safety of fremanezumab in a subgroup of participants $\geq 60$ years of age. Efficacy outcomes in the overall pooled population are also presented here for comparison.

Outcome measures assessed in participants $\geq 60$ years included the following: mean change from baseline (28-day pretreatment period) in the monthly average number of migraine days during the 12-week treatment period; monthly average number of headache days of at least moderate severity during the 12-week treatment period; monthly average number of days of any acute headache medication use; and weekly average number of migraine days during the first 4 weeks of treatment.

For this subgroup of participants $\geq 60$ years, the following outcomes were also assessed:

- Proportion of participants with $\geq 50 \%$ reduction from baseline (28-day pretreatment period) in the monthly average number of migraine days during the 12-week treatment period

- Mean change from baseline (Day 0) in scores on the 6-item Headache Impact Test (HIT-6; scores range from 36 to 78, with higher scores indicating greater impact of headache on functional status and wellbeing) [27] at 4 weeks after the last dose of study drug

- Mean change from baseline (Day 0) in scores on the Migraine Disability Assessment (MIDAS; scores range from 0 to 270 , with higher scores indicating more severe disability) $[28,29]$

- Mean change from baseline (Day 0) in domain scores on the Migraine-Specific Quality of Life (MSQoL) questionnaire (domains assessed: role function-restrictive [RFR; 7 items on how migraine limits daily activities], role function-preventive [RFP, 4 items on how migraine prevents these activities], emotional function [EF; 3 items on the emotional effects of migraine] [30]; scores range from 0 to 100, with higher scores indicating better health-related quality of life) at 4 weeks after the last dose of study drug

- Proportion of participants classified as responders on the Patient Global Impression of Change (PGIC; responder defined as an individual who reported a 
rating of 5-7 [moderately better, better, or a great deal better] on the PGIC)

- Change from baseline (Day 0) in domain scores on the Work Productivity and Activity Impairment (WPAI) assessment (percent work time missed due to health, percent impairment while working due to health, percent overall work impairment due to health, percent activity impairment due to health) [31]

Adverse events (AEs), serious AEs (SAEs), AEs leading to discontinuation, and CV AEs in participants with a CV medical history were also assessed.

\section{Statistical analyses}

Efficacy analyses were conducted in the full analysis set, which included all randomly assigned participants who received $\geq 1$ dose of study drug and had $\geq 10$ days of postbaseline efficacy assessments on the primary endpoint. Demographic and baseline characteristics were summarized descriptively. The mean change from baseline in the monthly average number of migraine days, weekly average number of migraine days, monthly average number of headache days of at least moderate severity, monthly average number of days of acute medication use, and MSQoL domain scores were analyzed using a mixed-effects model for repeated measures (MMRM) with treatment, sex or gender, study, region, month, and treatment-by-month as fixed effects and baseline value and years since onset of migraine as covariates. Mean changes from baseline in the monthly average number of migraine days and the monthly average number of headache days of at least moderate severity were evaluated separately for people with CM and EM. Mean changes from baseline in WPAI domain scores, HIT-6 scores, and MIDAS scores were evaluated using an analysis of covariance model with treatment, sex or gender, study, and region as fixed effects and baseline score and years since onset of migraine as covariates. The proportion of participants with $\mathrm{a} \geq 50 \%$ reduction in the monthly average number of migraine days was evaluated using a logistic regression model with treatment, sex or gender, and region as effects. Participants who discontinued early were considered non-responders for the overall analysis and each month following discontinuation. PGIC responder rates were evaluated using a CochranMantel-Haenszel (CMH) test stratified by study.

Adverse events, SAEs, AEs leading to discontinuation, and CV AEs in participants with a CV medical history were summarized descriptively.

\section{Data availability}

Anonymized data will be shared upon request from any qualified investigator.

\section{Results}

\section{Participants}

A total of 2843 participants were enrolled across all 3 studies (HALO CM, $N=1130$; HALO EM, $N=875$; FOCUS, $N=838$ ). The overall pooled full analysis population included 2823 participants (placebo, $n=939$; quarterly fremanezumab, $\mathrm{n}=939$; monthly fremanezumab, $n=945)$. Of these, $246(8.7 \%)$ participants were $\geq 60$ years of age and were included in these analyses (placebo, $n=80$; quarterly fremanezumab, $n=74$; monthly fremanezumab, $n=92$ ). Demographics and baseline characteristics for participants $\geq 60$ years were similar across treatment groups. The mean age ranged from approximately 63 to 64 years, $81 \%$ to $84 \%$ of participants were female, and the mean time since initial migraine diagnosis was approximately 37 to 38 years (Table 1 ). Chronic migraine (60\%) was more common in this pooled subpopulation aged $\geq 60$ years than EM (40\%). As expected, time since initial migraine diagnosis was longer in the subgroup of participants aged $\geq 60$ years than in the overall pooled population due to this subpopulation's advanced age. For participants with a CV medical history at baseline, the most commonly reported types of CV medical history were hypertension (50\%-71\%) and varicose vein $(0 \%-10 \%$; Additional file 1$)$.

\section{Monthly migraine days, monthly headache days of at least moderate severity, and days of acute medication use}

Among participants aged $\geq 60$ years in the pooled analysis, fremanezumab treatment resulted in significantly greater reductions from baseline during 12 weeks of double-blind treatment in the monthly average number of migraine days compared with placebo (least-squares mean [LSM (standard error [SE])] change from baseline: placebo, - 2.3 [0.57]; quarterly fremanezumab, - 4.3 [0.59]; monthly fremanezumab, -4.6 [0.54], both $P<0.01$ vs placebo; Fig. 1A) Compared with placebo, reductions from baseline during 12 weeks of double-blind treatment in monthly average migraine days were also shown to be significantly greater with both quarterly and monthly fremanezumab in participants aged $\geq 60$ years with EM $(P<0.05$ for both quarterly and monthly fremanezumab vs placebo) and with monthly fremanezumab in participants aged $\geq 60$ years with $\mathrm{CM} \quad(P<0.01$ for monthly fremanezumab vs placebo. Fig. 1B).

During the first 4 weeks of the double-blind treatment period in participants aged $\geq 60$ years, the reduction from baseline in the weekly average number of migraine days was significantly greater with fremanezumab compared with placebo at each weekly time point from Week 1 (LSM [SE] change from baseline: placebo, - 0.4 [0.20]; quarterly fremanezumab, - 1.1 [0.21]; monthly fremanezumab, -1.1 [0.19], both $P<0.01$ vs placebo) through 
Table 1 Demographics and baseline characteristics of participants $\geq 60$ years of age and overall population

\begin{tabular}{|c|c|c|c|c|c|c|}
\hline & \multicolumn{3}{|c|}{ Overall pooled population } & \multicolumn{3}{|c|}{ Participants $\geq 60$ years of age } \\
\hline & $\begin{array}{l}\text { Quarterly } \\
\text { fremanezumab } \\
n=943\end{array}$ & $\begin{array}{l}\text { Monthly } \\
\text { fremanezumab } \\
n=954\end{array}$ & $\begin{array}{l}\text { Placebo } \\
n=945\end{array}$ & $\begin{array}{l}\text { Quarterly } \\
\text { fremanezumab } \\
n=74\end{array}$ & $\begin{array}{l}\text { Monthly } \\
\text { fremanezumab } \\
n=92\end{array}$ & $\begin{array}{l}\text { Placebo } \\
n=80\end{array}$ \\
\hline Age, years, mean (SD) & $42.8(11.83)$ & $43.0(12.09)$ & $42.9(12.02)$ & $63.5(2.96)$ & $63.2(3.15)$ & $64.0(2.83)$ \\
\hline Female, n (\%) & $811(86)$ & $813(85)$ & $808(86)$ & $60(81)$ & $77(84)$ & $67(84)$ \\
\hline White, n (\%) & 787 (83) & $804(84)$ & 787 (83) & $71(96)$ & $81(88)$ & $70(88)$ \\
\hline $\mathrm{BMI}, \mathrm{kg} / \mathrm{m}^{2}$, mean (SD) & $26.3(4.98)$ & $26.0(4.94)$ & $26.4(4.81)$ & $25.5(4.14)$ & $25.7(4.18)$ & $26.0(4.01)$ \\
\hline \multicolumn{7}{|l|}{ Migraine classification, n (\%) } \\
\hline Chronic migraine & $545(58)$ & $553(58)$ & $541(57)$ & $56(76)$ & $46(50)$ & $45(56)$ \\
\hline Episodic migraine & $398(42)$ & $401(42)$ & $404(43)$ & $18(24)$ & $46(50)$ & $35(44)$ \\
\hline $\begin{array}{l}\text { Time since initial migraine } \\
\text { diagnosis, years, mean (SD) }\end{array}$ & $21.1(12.77)$ & $21.5(12.88)$ & $21.2(12.92)$ & $37.3(13.06)$ & $38.0(12.31)$ & $37.1(12.49)$ \\
\hline
\end{tabular}

$\mathrm{SD}$, standard deviation; BMI, body mass index

Week 4 (placebo, - 0.5 [0.19]; quarterly fremanezumab, - $1.2[0.20], P<0.05$ vs placebo; monthly fremanezumab, -1.2 [0.18], $P<0.01$ vs placebo; Fig. 2A).

In this pooled analysis, treatment with fremanezumab resulted in a significantly greater reduction in the monthly

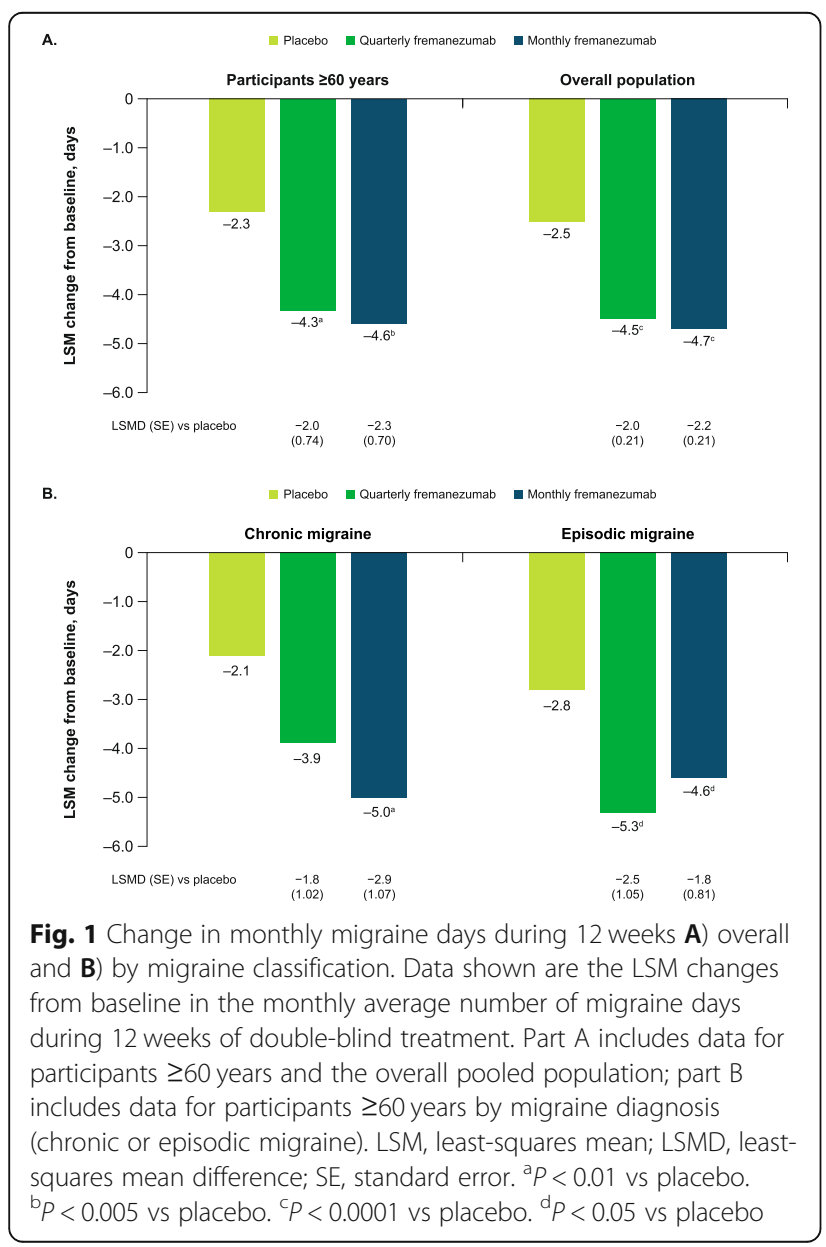

average number of headache days of at least moderate severity compared with placebo during 12 weeks of treatment in participants aged $\geq 60$ years (LSM [SE] change from baseline: placebo, -2.1 [0.53]; quarterly fremanezumab, -3.9 [0.55], $P<0.05$ vs placebo; monthly fremanezumab, -4.2 [0.51], $P<0.01$ vs placebo; Fig. 3A). Reductions in monthly average headache days of at least moderate severity from baseline during 12 weeks of double-blind treatment were significantly greater with quarterly and monthly fremanezumab compared with placebo in participants aged $\geq 60$ years with EM $(P<0.05$ for both quarterly and monthly fremanezumab vs placebo) and with monthly fremanezumab compared with placebo in the $\mathrm{CM}$ subset $(P<0.01$ for monthly fremanezumab vs placebo; Fig. 3B).

Among participants $\geq 60$ years of age, the proportion of participants with $\geq 50 \%$ reduction in the monthly average number of migraine days during 12 weeks of treatment was significantly greater with monthly fremanezumab compared with placebo (placebo, 25\%; quarterly fremanezumab, $36 \%, P=0.12$ vs placebo; monthly fremanezumab, $40 \%, P<0.05$ vs placebo; Fig. 4 ).

During 12 weeks of treatment, quarterly and monthly fremanezumab treatment resulted in significantly greater reductions from baseline in the monthly average number of days of acute medication use compared with placebo in participants aged $\geq 60$ years (LSM [SE] change from baseline: placebo, -1.3 [0.55]; quarterly fremanezumab, - $3.7[0.56], P<0.001$ vs placebo; monthly fremanezumab, -4.0 [0.52], $P=0.0001$ vs placebo; Fig. 5).

These reductions in the monthly average number of migraine days, weekly average number of migraine days, monthly average number of headache days of at least moderate severity, and monthly average number of days of acute medication use for participants aged $\geq 60$ years were comparable to those for the overall pooled population (Figs. 1, 2B, 3, 4, and 5). 


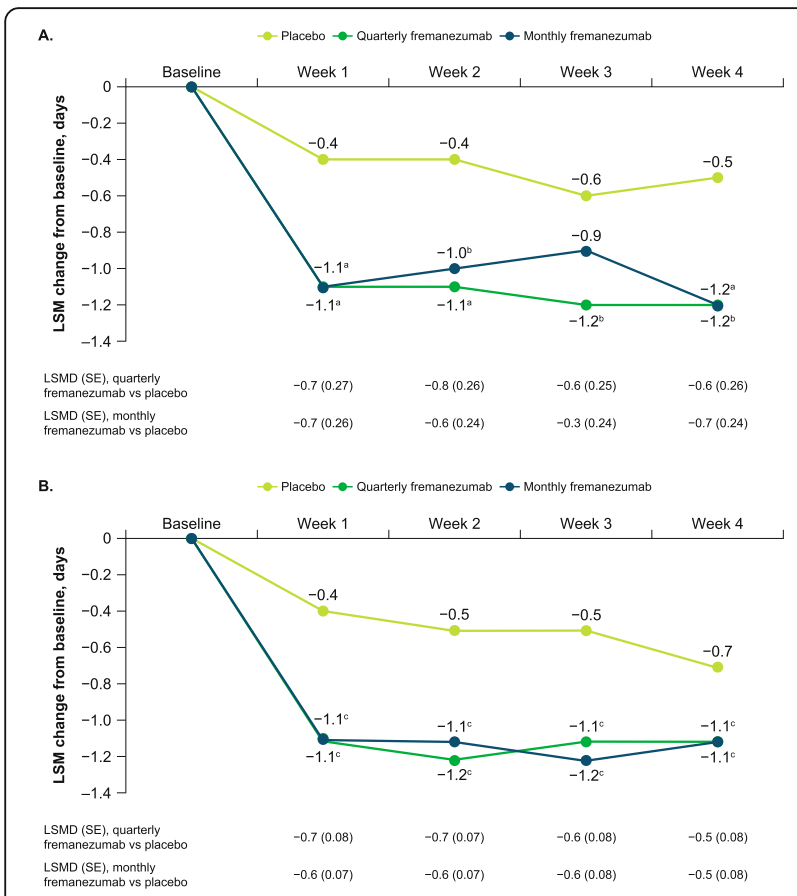

Fig. 2 Change in weekly migraine days in A) participants aged $\geq 60$ years and $\mathbf{B}$ ) overall population. LSM, least-squares mean; LSMD, least-squares mean differences; SE, standard error. Data shown are the LSM changes from baseline in the weekly average number of migraine days over the first 4 weeks of double-blind treatment. ${ }^{\mathrm{a}} P<0.01$ vs placebo. ${ }^{\mathrm{b}} P<0.05$ vs placebo. ${ }^{\mathrm{c}} P<0.0001$ vs placebo

\section{Disability and quality-of-life outcomes}

During the 12-week treatment period, reductions from baseline in HIT- 6 scores in participants aged $\geq 60$ years were greater with fremanezumab versus placebo, with significant differences for monthly fremanezumab compared with placebo (LSM [SE] change from baseline: placebo, - 2.7 [0.92]; quarterly fremanezumab, - 4.3 [0.89], $P=0.1585$ vs placebo; monthly fremanezumab, -6.8 [0.94], $P=0.0005$ vs placebo; Fig. 6 A). The LSM difference (SE) in the change in HIT-6 score for monthly fremanezumab versus placebo (-4.2 [1.17]) met the 2.3point criterion for a clinically meaningful improvement [32].

Reductions from baseline in MIDAS scores during 12 weeks of treatment in participants aged $\geq 60$ years were also greater with both fremanezumab dosing regimens compared with placebo, with a significant difference for monthly fremanezumab compared with placebo (placebo, - 11.0 [3.85]; quarterly fremanezumab, -21.7 [4.37], $P=0.0506$ vs placebo; monthly fremanezumab, -24.0 [3.56], $P<0.01$ vs placebo; Fig. 6B).

MSQoL scores improved from baseline in participants aged $\geq 60$ years with both fremanezumab dosing regimens across all domains, including the RFR score and EF scores (Fig. 7A). These improvements in MSQoL scores at 3 months were significantly greater with both

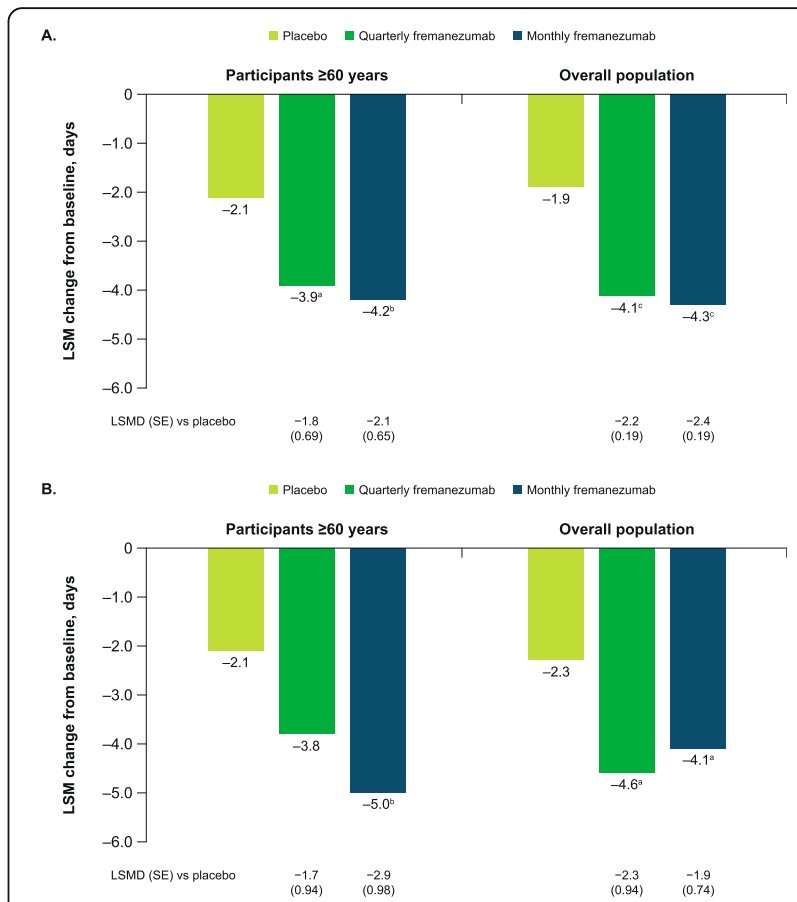

Fig. 3 Change in monthly headache days during 12 weeks $\mathbf{A}$ ) overall and $\mathbf{B}$ ) by migraine classification. LSM, least-squares mean; LSMD, least-squares mean difference; SE, standard error. Data shown are the LSM changes from baseline in the monthly average number of headache days of at least moderate severity during 12 weeks of double-blind treatment. Part A includes data for participants $\geq 60$ years and the overall pooled population; part $B$ includes data for participants $\geq 60$ years by migraine diagnosis (chronic or episodic migraine). ${ }^{\mathrm{a}} P<0.05$ vs placebo. ${ }^{\mathrm{b}} p<0.005$ vs placebo. ${ }^{c} p<0.0001$ vs placebo

fremanezumab dosing regimens compared with placebo for participants aged $\geq 60$ years for the RFR and EF scores (all $P<0.05$ vs placebo).

Improvements in all WPAI domain scores were observed in participants aged $\geq 60$ years with

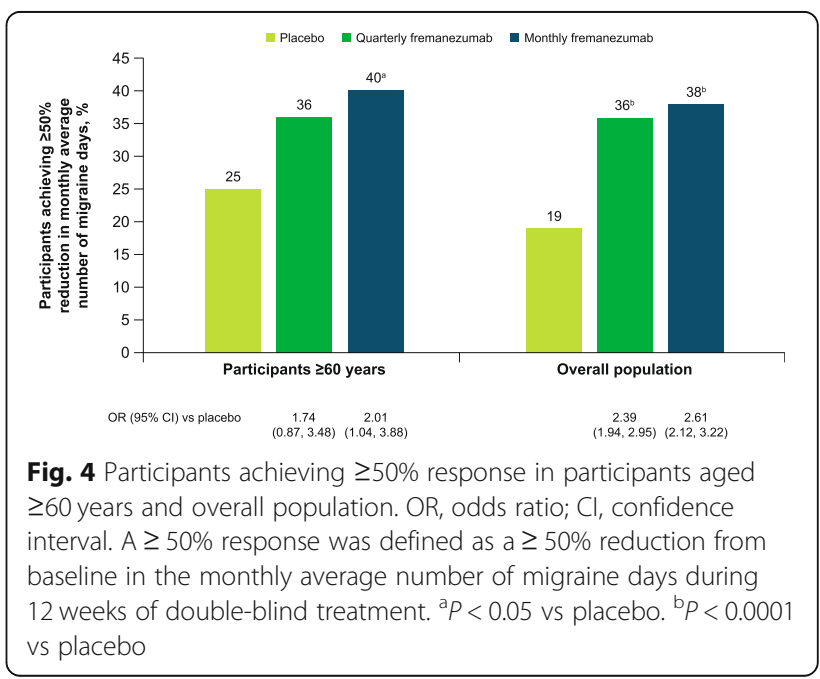




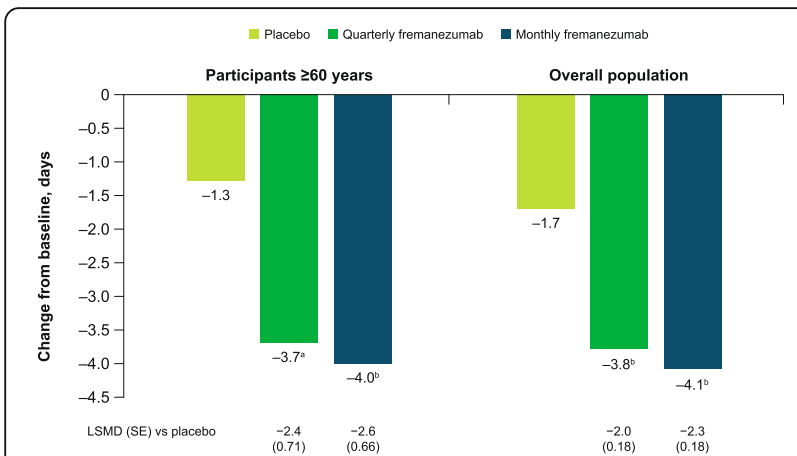

Fig. 5 Change in monthly days of acute medication use in participants aged $\geq 60$ years and overall population. LSM, leastsquares mean; LSMD, least-squares mean difference; SE, standard error. Data shown are the LSM changes from baseline in the monthly average number of days of acute headache medication use during 12 weeks of double-blind treatment. ${ }^{a} P<0.001$ vs placebo. ${ }^{\mathrm{b}} P \leq 0.0001$ vs placebo

fremanezumab treatment (Fig. 8A). Compared with placebo, improvements in WPAI scores were significantly greater for the percent impairment while working domain score for both fremanezumab dosing regimens, for the percent overall work impairment domain score for quarterly fremanezumab, and for the percent

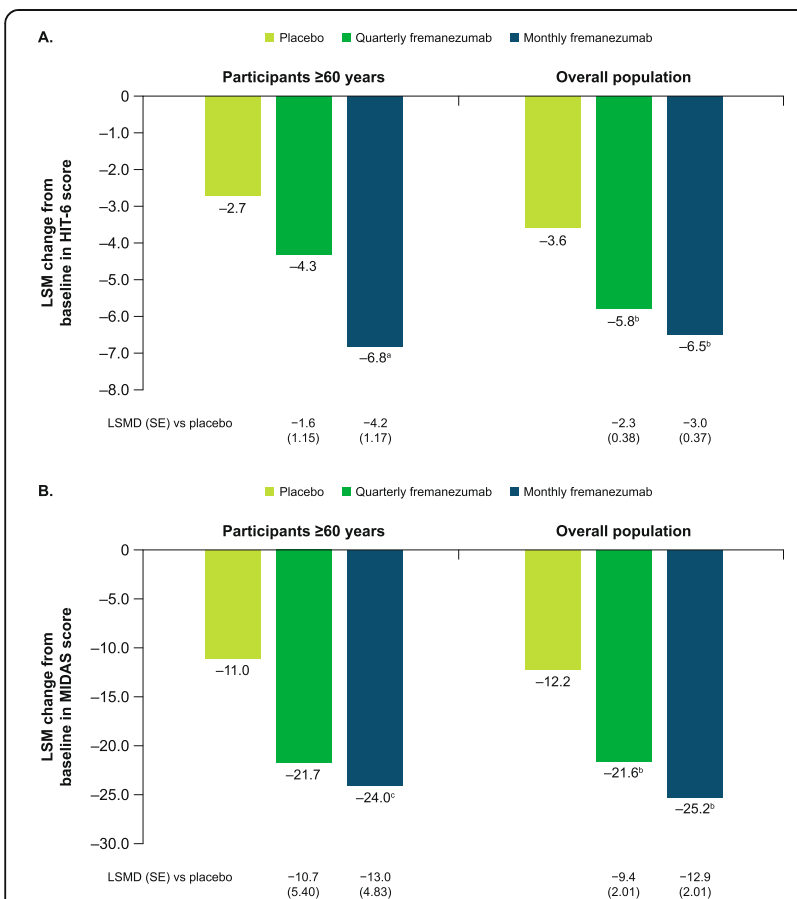

Fig. 6 Change in A) HIT-6 and B) MIDAS scores in participants aged $\geq 60$ years and overall population. HIT-6, 6-item Headache Impact Test; MIDAS, Migraine Disability Assessment; LSM, least-squares mean; LSMD, least-squares mean difference; SE, standard error. Data shown are the LSM changes from baseline in HIT-6 and MIDAS scores during 12 weeks of double-blind treatment. ${ }^{\mathrm{a}} P=0.0005$ vs placebo. ${ }^{b} P<0.0001$ vs placebo. ${ }^{c} P<0.01$ vs placebo activity impairment score for monthly fremanezumab (all $P<0.05$ vs placebo).

The proportion of participants aged $\geq 60$ years who were classified as responders on the PGIC scale (score $\geq 5$ ) was significantly higher with both fremanezumab dosing regimens compared with placebo (both $P<0.01$; Fig. 9).

Improvements in HIT-6, MIDAS, MSQoL, WPAI, and PGIC scores for the subgroup of participants aged $\geq 60$ years were comparable to those in the overall pooled population (Figs. 6, 7B, 8B, and 9).

\section{Safety}

This pooled analysis of AEs reported in a subgroup of participants aged $\geq 60$ years showed that fremanezumab was generally safe and well tolerated. In the overall subgroup of participants $\geq 60$ years of age, AEs were reported for similar proportions of participants across treatment groups. Serious AEs (all treatment groups, $3 \%$ ) and AEs leading to discontinuation (quarterly fremanezumab, $1 \%$; monthly fremanezumab, $1 \%$; placebo, $3 \%)$ were infrequent in participants receiving fremanezumab and comparable to placebo. The most common AEs were injection-site induration, injection-site pain, and injection-site erythema (Table 2). Among participants aged $\geq 60$ years with a CV medical history, CV AEs occurred in a similar proportion of participants with or without a CV medical history (Table 3). There was only one SAE reported in participants aged $\geq 60$ years with a $\mathrm{CV}$ medical history, and none reported in participants aged $\geq 60$ years without a CV medical history. These safety results are comparable to those in the overall pooled population (any AE, quarterly fremanezumab [65\%], monthly fremanezumab [62\%], placebo [58\%]; SAEs, quarterly fremanezumab $[<1 \%]$, monthly fremanezumab [1\%], placebo [2\%]; AEs leading to discontinuation, quarterly fremanezumab [1\%], monthly fremanezumab [2\%], placebo [2\%]; any CV AE with $\mathrm{CV}$ medical history, quarterly fremanezumab [4\%], monthly fremanezumab [6\%], placebo [4\%]).

\section{Discussion}

As compared with placebo, fremanezumab treatment resulted in reductions of approximately 2 days from baseline in the monthly average number of migraine days and in the monthly average number of headache days of at least moderate severity over 12 weeks in this subgroup of participants $\geq 60$ years of age. Reductions in migraine days were seen as early as Week 1 with fremanezumab treatment, and consistent significant reductions were observed with both fremanezumab dosing regimens compared with placebo during each of the first 4 weeks of treatment. The proportion of participants achieving clinically meaningful response rates $(\geq 50 \%$ reduction in monthly average number of migraine days) with 
A.

Placebo Quarterly fremanezumab $\quad$ Monthly fremanezumab

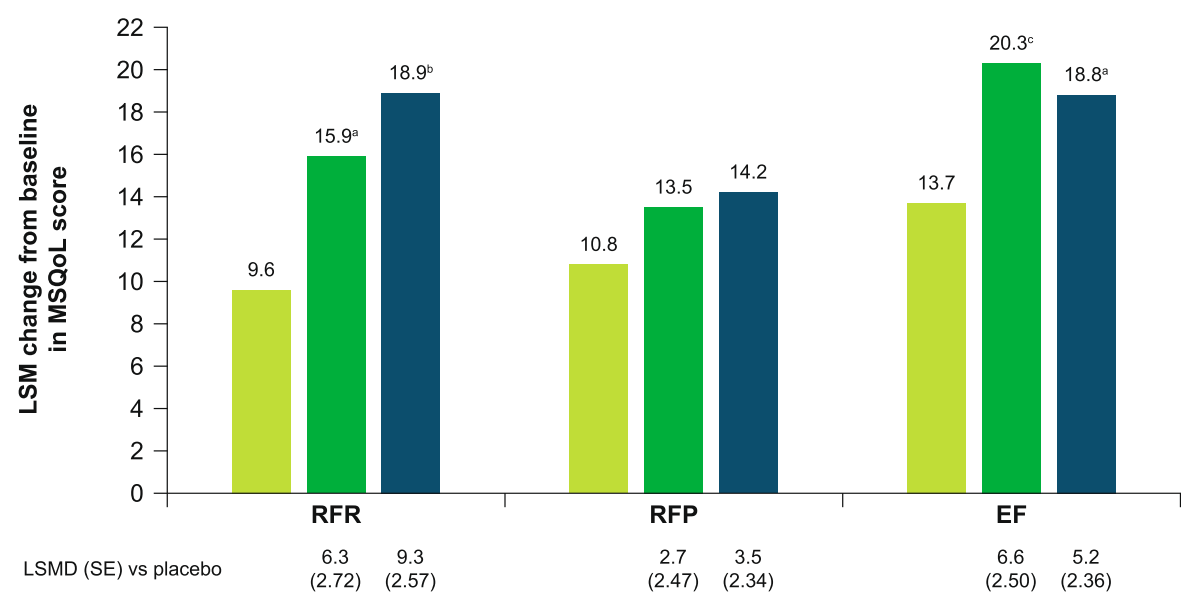

B.

Placebo Quarterly fremanezumab Monthly fremanezumab

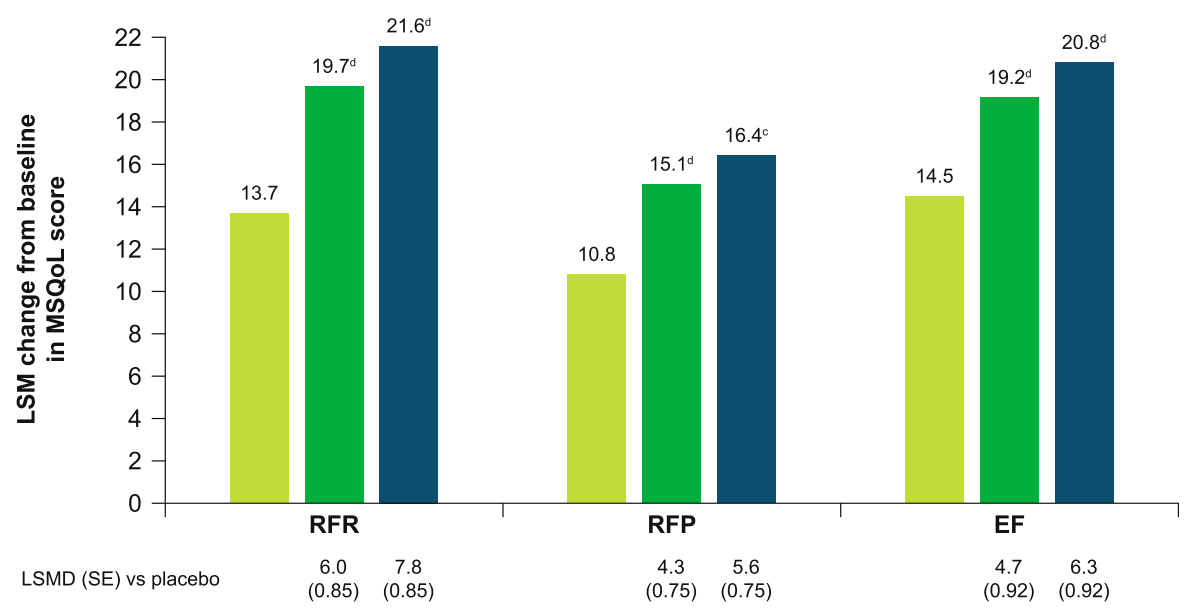

Fig. 7 Change in MSQoL domain scores in A) participants aged $\geq 60$ years and B) overall population. MSQoL, Migraine-specific Quality of Life; $R F R$, role-function restrictive; RFP, role-function preventive; EF, emotional function; LSM, least-squares mean; LSMD, least-squares mean difference; $\mathrm{SE}$, standard error. Data shown are the LSM changes from baseline in MSQoL domain scores at Week $12 .{ }^{\mathrm{a}} P<0.05$ vs placebo. ${ }^{\mathrm{b}} P<0.0005$ vs placebo. ${ }^{c} P<0.01$ vs placebo. ${ }^{d} p<0.0001$ vs placebo

fremanezumab was $11 \%$ higher with quarterly dosing and $15 \%$ higher with monthly dosing compared with placebo; the difference between fremanezumab and placebo was statistically significant for monthly fremanezumab, but not quarterly fremanezumab. Additionally, over 12 weeks of fremanezumab treatment, participants aged $\geq 60$ years experienced an approximately 2.5 -day per month greater reduction in days of acute headache medication use than with placebo. These results were comparable to those observed in the overall pooled population.

In participants aged $\geq 60$ years, treatment with quarterly or monthly fremanezumab over 12 weeks resulted in clinically meaningful improvements in a variety of outcomes related to quality of life. Participants receiving fremanezumab experienced significantly greater improvements in headache-related disability, based on reductions in MIDAS and HIT-6 scores, as compared with participants who received placebo. Improvements from baseline versus placebo were observed with fremanezumab in all MSQoL and WPAI domains. The proportion of participants categorized as PGIC responders was also significantly greater with fremanezumab versus placebo.

Although migraine treatment may be more difficult in older patients [5, 7], fremanezumab showed comparable, or for some endpoints numerically better, efficacy in the subgroup of participants aged $\geq 60$ years and in the overall pooled population. For example, for the WPAI domain of work time missed, fremanezumab showed numerically greater improvements from baseline in the 
A.

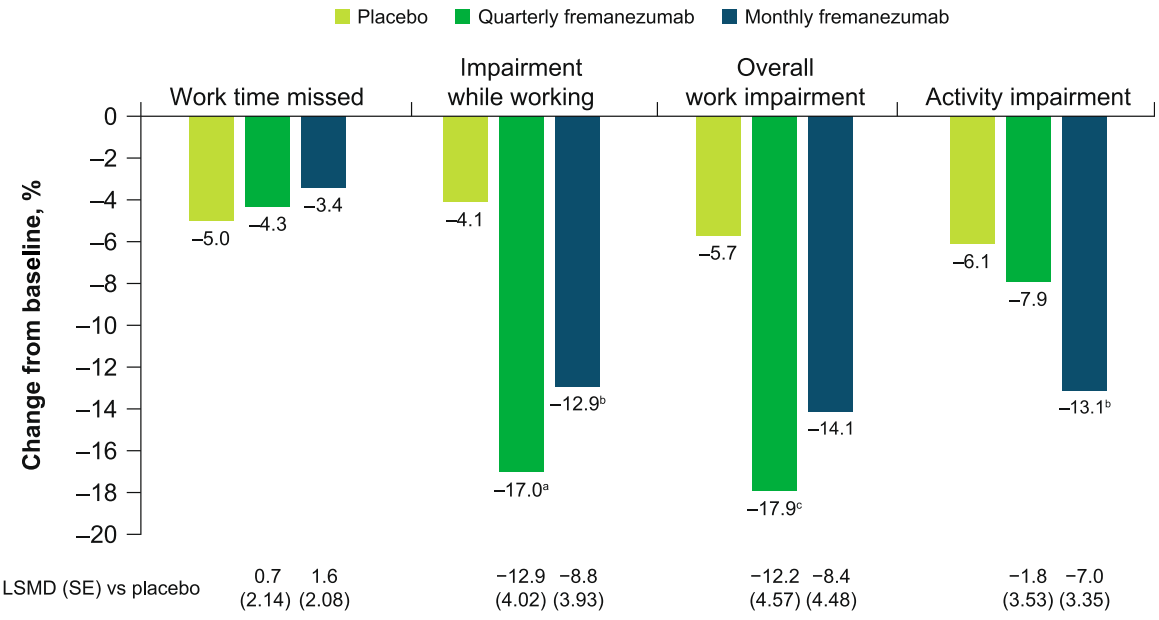

B.

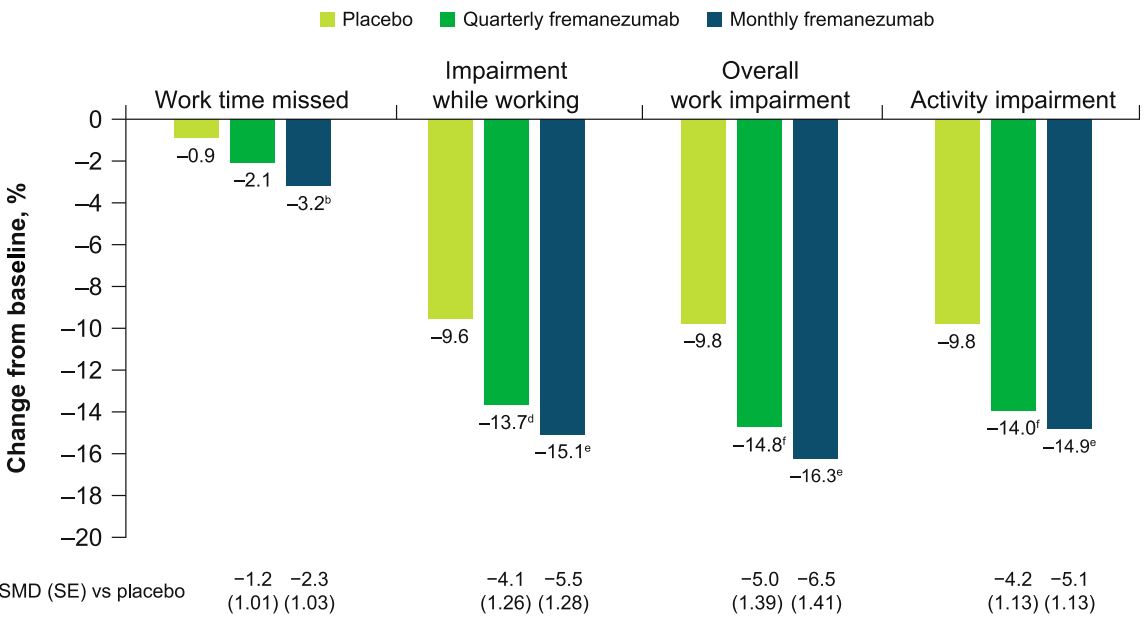

Fig. 8 Change in WPAl domain scores in A) participants aged $\geq 60$ years and $\mathbf{B}$ ) overall population. WPAl, Work Productivity and Activity Impairment; LSM, least-squares mean; LSMD, least-squares mean difference; SE, standard error. Data shown are the LSM changes from baseline in WPAl domain scores during 12 weeks of double-blind treatment. ${ }^{a} P<0.005$ vs placebo. ${ }^{b} p<0.05$ vs placebo. ${ }^{c} P<0.01$ vs placebo. ${ }^{d} p \leq 0.001$ vs placebo. ${ }^{e} P<0.0001$ vs placebo. ${ }^{f} P<0.0005$ vs placebo

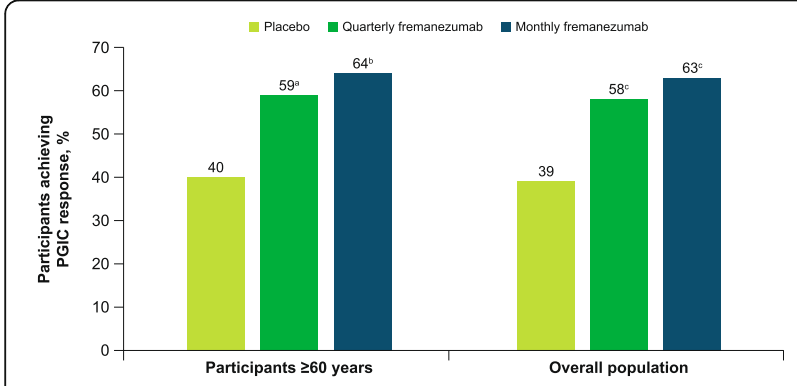

Fig. 9 Proportion of PGIC responders in participants aged $\geq 60$ years and the overall population. PGIC, patient global impression of change. Data shown are the proportion of PGIC responders (defined as individuals who reported a rating of 5-7 [moderately better, better, or a great deal better] on the PGIC) at Week 12. ${ }^{\mathrm{a}} P<0.01$ vs placebo. ${ }^{b} P<0.005$ vs placebo. ${ }^{c} P<0.0001$ vs placebo subgroup aged $\geq 60$ years compared with the overall population. This consistency of the efficacy of fremanezumab in this subgroup of participants aged $\geq 60$ years with that in the overall pooled population provides evidence that fremanezumab is effective in reducing migraine in older individuals, including those with difficultto-treat migraine.

Determining an optimal migraine preventive pharmacotherapy plan for older patients can be challenging due to the traditionally lower participation rates by older people with migraine, as well as restrictive exclusion criteria in most migraine clinical trials [5]. The safety profile for fremanezumab in participants aged $\geq 60$ years is consistent across treatment groups and is comparable to the overall pooled population. Generally, AEs were infrequent in older participants with a CV medical history. Of note, considering the higher risk for $\mathrm{CV}$ disease and 
Table 2 Overall AEs in participants $\geq 60$ years of age with $\geq 5 \%$ incidence in any treatment group

\begin{tabular}{llll}
\hline AEs, $\boldsymbol{n}(\mathbf{\%})$ & $\begin{array}{l}\text { Quarterly fremanezumab } \\
(\boldsymbol{n = 7 4 )}\end{array}$ & $\begin{array}{l}\text { Monthly fremanezumab } \\
(\boldsymbol{n = 9 2 )}\end{array}$ & $\begin{array}{l}\text { Placebo } \\
(\boldsymbol{n}=\mathbf{8 0})\end{array}$ \\
\hline Any AE & $48(65)$ & $53(58)$ & $44(55)$ \\
SAEs & $2(3)$ & $3(3)$ & $2(3)$ \\
AEs leading to discontinuation & $1(1)$ & $1(1)$ & $2(3)$ \\
Most common AEs (incidence $\geq 5 \%$ in any treatment group) & $23(25)$ & $19(21)$ & $14(18)$ \\
Injection-site induration & $11(15)$ & $10(11)$ & $10(13)$ \\
Injection-site pain & $13(18)$ & $5(5)$ & $4(5)$ \\
Injection-site erythema & $8(11)$ &
\end{tabular}

$\mathrm{AE}$, adverse event; $\mathrm{SAE}$, serious adverse event

other comorbidities among older individuals [9, 33], rates of $\mathrm{CV}$ AEs were low, even in participants with a $\mathrm{CV}$ medical history. Further, there were no SAEs reported in this subgroup.

Whereas migraine is more common in individuals younger than 55 years, a considerable proportion of people older than this also experience migraine [7, 9, 33]. Many cross-sectional studies have shown that migraine persists in people aged $\geq 60$ years, with a prevalence of approximately $5 \%$ even in those older than 75 years [34]. Currently, there are no subgroup analyses for other mAbs targeting the CGRP ligand that examined efficacy in older study participants with migraine. Erenumab is the only other CGRP pathwaytargeted $\mathrm{mAb}$ (receptor targeted) that has analyzed safety and tolerability in subgroups of older study participants (50-55 and $>55$ years of age) with migraine. As with fremanezumab, treatment-emergent $\mathrm{AE}$ rates were comparable for the erenumab treatment groups and the placebo group in these older subgroups [35]. Rates of SAEs and AEs leading to discontinuation were also infrequent and similar across the erenumab and placebo groups for these older participants [35].

The results of this subgroup analysis may be subject to limitations. Although this analysis included participants from 3 large studies, there were relatively small numbers of participants $\geq 60$ years in each group. The lower number of participants may have limited the ability to detect significant between-group differences for all endpoints (eg, the $\geq 50 \%$ response rate for change in monthly migraine days for quarterly fremanezumab versus placebo). However, the results reported here may be generalizable because the efficacy and safety results from the subgroup were comparable to the overall pooled population. Additionally, of 7171 participants screened in the HALO and FOCUS studies, 610 met the exclusion criteria, which included a history of clinically significant $\mathrm{CV}$ disease as determined by the investigator, so the participants included in these analyses likely have a milder CV medical history than older people with migraine in the general population.

As mentioned, older individuals with migraine likely have a range of comorbidities, including hypertension,

Table 3 Cardiovascular adverse events in participants $\geq 60$ years of age with a cardiovascular medical history

\begin{tabular}{|c|c|c|c|c|}
\hline \multirow[t]{2}{*}{ CV AEs, n (\%) } & \multirow{2}{*}{$\frac{\text { Quarterly fremanezumab }}{\text { (CM/EM: } 675 \mathrm{mg} / \mathrm{PBO} / \mathrm{PBO})}$} & \multicolumn{2}{|l|}{ Monthly fremanezumab } & \multirow[t]{2}{*}{ Placebo } \\
\hline & & (EM: $225 / 225 / 225 \mathrm{mg}$ ) & (CM: 675/225/225 mg) & \\
\hline Participants with CV medical history & $(n=30)$ & $(n=6)$ & $(n=14)$ & $(n=23)$ \\
\hline$\geq 1 \mathrm{CV} A E$ & $1(3)$ & 0 & $2(14)$ & $1(4)$ \\
\hline \multicolumn{5}{|c|}{ AEs with incidence $\geq 1$ participant in any treatment/dose group } \\
\hline Palpitations & 0 & 0 & $1(7)$ & $1(4)$ \\
\hline Hypertensive crisis & 0 & 0 & $1(7)$ & 0 \\
\hline Supraventricular tachycardia & $1(3)$ & 0 & 0 & 0 \\
\hline Participants without CV medical history & $(n=44)$ & $(n=40)$ & $(n=32)$ & $(n=57)$ \\
\hline$\geq 1 \mathrm{CV} A \mathrm{E}$ & 0 & 0 & $2(6)$ & 0 \\
\hline \multicolumn{5}{|c|}{ AEs with incidence $\geq 1$ participant in any treatment/dose group } \\
\hline Hot flush & 0 & 0 & $1(3)$ & 0 \\
\hline Hypertension & 0 & 0 & $1(3)$ & 0 \\
\hline
\end{tabular}

$\mathrm{CM}$, chronic migraine; $\mathrm{EM}$, episodic migraine; $\mathrm{PBO}$, placebo; $\mathrm{CV}$, cardiovascular; $\mathrm{AE}$, adverse event 
heart disease, or psychiatric disorders, that also influence management of their migraine [7, 33]. Even in a complex population, effective migraine management may improve their quality of life $[7,33]$. With a favorable safety profile, these results demonstrate that fremanezumab may be an effective migraine preventive treatment option for older individuals with difficult-to-treat disease.

\section{Conclusions}

This pooled subgroup analysis demonstrated that fremanezumab treatment was effective in reducing migraine days, headache days of at least moderate severity, and days of acute medication use over 12 weeks of treatment in participants aged $\geq 60$ years with EM or CM. Additionally, fremanezumab improved quality of life and headache-related disability in this older population. The consistent efficacy and safety results of fremanezumab across treatment groups in this subgroup analysis demonstrate that fremanezumab is an effective preventive treatment option for individuals $\geq 60$ years of age, including those with comorbidities and difficult-to-treat EM or CM.

\begin{abstract}
Abbreviations
CGRP: calcitonin gene-related peptide; CM: chronic migraine; CV: cardiovascular; ECG: electrocardiogram; EM: episodic migraine: mAbs: monoclonal antibodies; ICHD: International Classification of Headache Disorders; HIT-6: 6-item Headache Impact Test; MIDAS: Migraine Disability Assessment; RFP: role function-preventive; RFR: role function-restrictive; EF: emotional function; WPAl: Work Productivity and Activity Impairment; PGIC: Patient Global Impression of Change; AE: adverse event; SAE: serious adverse event; LSM: least-squares mean; LSMD: least-squares mean difference; OR: odds ratio; BMI: body mass index; PBO: placebo
\end{abstract}

\section{Supplementary Information}

The online version contains supplementary material available at https://doi. org/10.1186/s10194-021-01351-2.

Additional file 1. Types of CV Medical History at Baseline.

\section{Acknowledgments}

We thank the individuals who participated in these studies and their families, all investigators, site personnel, and the coordinating investigators. Editorial assistance was provided by Alyssa Nguyen, PharmD, of Cello Health Communications/MedErgy. This assistance was in accordance with Good Publication Practice (CPP3) guidelines and funded by Teva Pharmaceuticals. The authors maintained full editorial control of the manuscript and decision to submit for publication.

\section{Authors' contributions}

S.J.N., S.N., J.M.C., X.N., L.J., V.R.C., L.J.K., D. H-L., D.K., and C.L. contributed to drafting of the manuscript, critically revising of the manuscript for important intellectual concepts. All authors read and approved the final manuscript.

\section{Funding}

This study was funded by Teva Pharmaceuticals Ltd., Petach Tikva, Israel. Named authors who are or were employees of Teva Pharmaceuticals participated in the interpretation of data and writing of the manuscript.

\section{Availability of data and materials}

Anonymized data, as described in this manuscript, will be shared upon request from any qualified investigator by the author investigators or Teva Pharmaceuticals Industries, Ltd.

\section{Declarations}

Ethics approval and consent to participate

As part of the original study, all participants provided written informed consent, and the trial was approved by all relevant review bodies. Because the subgroup analyses used existing data from the primary study, additional consent was not required.

\section{Consent for publication}

Not applicable.

\section{Competing interests}

S.J.N. has received honoraria for consulting from Allergan/AbbVie, Amgen/ Novartis, Axsome, BioDelivery Sciences, Biohaven, Eli Lilly, Fenix Group International, Impel, Nesos Corp (formerly Vorso Corp), and Teva Pharmaceuticals. S.J.N. has received honoraria for speaking from Allergan/ AbbVie, Amgen/Novartis, Eli Lilly, and Teva Pharmaceuticals. S.J.N. has received honoraria for work in education or publishing from the American Academy of Neurology, the American Headache Society, Evolve Med Ed, the Massachusetts Medical Society, MedLink Neurology, MJH Life Sciences, NACCME, Neurology Learning Network, Pennsylvania Neurologic Society, Springer, WebMD/Medscape, and Wolters-Kluwer. S.J.N. has received legal fees for serving as a medical expert to Jackson \& Campbell. J.M.C. is a former employee of Teva Pharmaceuticals. X.N., L.J., V.R.C., and L.J.K. are employees of Teva Pharmaceuticals. D.H.-L. has no competing interests. D.K. has served as an advisor to Alder, Amgen, and Eli Lilly and has received research support from Alder, Allergan, Amgen, CoLucid-Eli Lilly, Genentech, Teva Pharmaceuticals, VM Biopharma, and Zosano. C.L. has received personal fees from Allergan, Eli Lilly, Novartis, and Teva Pharmaceuticals; has participated in clinical trials as a principal investigator for Novartis and Teva Pharmaceuticals; and has no ownership interest and does not own stocks in any pharmaceutical company.

\section{Author details}

${ }^{1}$ Department of Neurology, Thomas Jefferson University, Philadelphia, PA, USA. ${ }^{2}$ Department of Neurology, University Hospital Halle (Saale) and University Halle-Wittenberg, Halle, Germany. ${ }^{3}$ Teva Pharmaceutical Industries, West Chester, PA, USA. ${ }^{4}$ Department of Neurology and Westgerman Headache Center Essen, University Hospital, Essen, Germany. ${ }^{5}$ California Medical Clinic for Headache, Santa Monica, CA, USA. ${ }^{6}$ Headache Medical Centre, Linz, Austria. ${ }^{7}$ Department of Neurology, Konventhospital Barmherzige Brüder, Linz, Austria.

Received: 9 September 2021 Accepted: 31 October 2021

Published online: 24 November 2021

\section{References}

1. Steiner TJ, Stovner LJ, Jensen R, Uluduz D, Katsarava Z, on behalf of Lifting the Burden: the Global Campaign Against Headache (2020) Migraine remains second among the world's causes of disability, and first among young women: findings from GBD2019. J Headache Pain 21(1):137. https:// doi.org/10.1186/s10194-020-01208-0

2. Vos T, Lim S, Abbafati C et al (2020) Global burden of 369 diseases and injuries in 204 countries and territories, 1990-2019: a systematic analysis for the global burden of disease study 2019. Lancet 396(10258):1204-1222. https://doi.org/10.1016/50140-6736(20)30925-9

3. Saylor D, Steiner TJ (2018) The global burden of headache. Semin Neurol 38(02):182-190. https://doi.org/10.1055/s-0038-1646946

4. Agosti R (2018) Migraine burden of disease: from the patient's experience to a socio-economic view. Headache 58:17-32. https://doi.org/10.1111/hea d.13301

5. Curto M, Capi M, Martelletti P, Lionetto L (2019) How do you choose the appropriate migraine pharmacotherapy for an elderly person? Expert Opin Pharmacother 20(1):1-3. https://doi.org/10.1080/14656566.2018.1543660

6. Hershey LA, Bednarczyk EM (2013) Treatment of headache in the elderly. Curr Treat Options Neurol 15(1):56-62. https://doi.org/10.1007/s11940-0120205-6

7. Starling AJ (2018) Diagnosis and management of headache in older adults. Mayo Clin Proc 93(2):252-262. https://doi.org/10.1016/j.mayocp.2017.12.002 
8. Wang SJ, Liu HC, Fuh JL, Liu CY, Wang PN, Lu SR (1999) Comorbidity of headaches and depression in the elderly. Pain 82(3):239-243. https://doi. org/10.1016/S0304-3959(99)00057-3

9. Wijeratne T, Tang HM, Crewther D, Crewther S (2019) Prevalence of migraine in the elderly: a narrated review. Neuroepidemiology 52(1-2): 104-110. https://doi.org/10.1159/000494758

10. Breslau N, Lipton RB, Stewart WF, Schultz LR, Welch KM (2003) Comorbidity of migraine and depression: investigating potential etiology and prognosis. Neurology 60(8):1308-1312. https://doi.org/10.1212/01.WNL.0000058907.41 080.54

11. Lampl C, Thomas H, Tassorelli C, Katsarava Z, Lainez JM, Lanteri-Minet M et al (2016) Headache, depression and anxiety: associations in the Eurolight project. J Headache Pain 17(1):59. https://doi.org/10.1186/s10194-016-0649-2

12. Antonaci F, Nappi G, Galli F, Manzoni GC, Calabresi P, Costa A (2011) Migraine and psychiatric comorbidity: a review of clinical findings. $J$ Headache Pain 12(2):115-125. https://doi.org/10.1007/s10194-010-0282-4

13. Buse DC, Silberstein SD, Manack AN, Papapetropoulos S, Lipton RB (2013) Psychiatric comorbidities of episodic and chronic migraine. J Neurol 260(8): 1960-1969. https://doi.org/10.1007/s00415-012-6725-x

14. Jackson JL, Cogbill E, Santana-Davila R, Eldredge C, Collier W, Gradall A, Sehgal N, Kuester J (2015) A comparative effectiveness meta-analysis of drugs for the prophylaxis of migraine headache. PLoS One 10(7):e0130733. https://doi.org/10.1371/journal.pone.0130733

15. Jackson JL, Kuriyama A, Hayashino Y (2012) Botulinum toxin a for prophylactic treatment of migraine and tension headaches in adults: a meta-analysis. JAMA 307(16):1736-1745. https:/doi.org/10.1001/jama.2012.505

16. Blumenfeld AM, Bloudek LM, Becker WJ, Buse DC, Varon SF, Maglinte GA, Wilcox TK, Kawata AK, Lipton RB (2013) Patterns of use and reasons for discontinuation of prophylactic medications for episodic migraine and chronic migraine: results from the second international burden of migraine study (IBMS-II). Headache 53(4):644-655. https://doi.org/10.1111/head.12055

17. Hepp Z, Dodick DW, Varon SF, Chia J, Matthew N, Gillard P, Hansen RN, Devine EB (2017) Persistence and switching patterns of oral migraine prophylactic medications among patients with chronic migraine: a retrospective claims analysis. Cephalalgia 37(5):470-485. https://doi.org/1 $0.1177 / 0333102416678382$

18. Martelletti P, Schwedt TJ, Lanteri-Minet M, Quintana R, Carboni V, Diener HC, Ruiz de la Torre E, Craven A, Rasmussen AV, Evans S, Laflamme AK, Fink R, Walsh D, Dumas P, Vo P (2018) My migraine voice survey: a global study of disease burden among individuals with migraine for whom preventive treatments have failed. J Headache Pain 19(1):115. https://doi.org/10.1186/s1 0194-018-0946-z

19. AIMOVIG (erenumab aooe) [prescribing information]. Amgen Inc, CA. Revised 2021. Available from: https://www.pi.amgen.com/ /media/amgen/ repositorysites/pi-amgen-com/aimovig/aimovig_pi_hcp_english.ashx. Accessed 7 Sept 2021

20. AJOVY (fremanezumab-vfrm) [prescribing information]. Teva Pharmaceuticals, PA. Revised 2021. Available from: https://www.ajovyhcp. com/globalassets/ajovy/ajovy-pi.pdf. Accessed 7 Sept 2021

21. EMGALITY (galcanezumab-gnlm) [prescribing information]. Eli Lilly and Company, IN. Revised 2019. Available from: http://uspl.lilly.com/emgality/ emgality.html\#pi. Accessed 7 Sept 2021

22. VYEPT|TM (eptinezumab-jimr) [prescribing information]. Lundbeck Seattle BioPharmaceuticals, Inc, WA. Revised 2020. Available from: https://www.a ccessdata.fda.gov/drugsatfda_docs/label/2020/761119s000lbl.pdf. Accessed 7 Sept 2021

23. Dodick DW, Silberstein SD, Bigal ME, Yeung PP, Goadsby PJ, Blankenbiller T, Grozinski-Wolff M, Yang R, Ma Y, Aycardi E (2018) Effect of fremanezumab compared with placebo for prevention of episodic migraine: a randomized clinical trial. JAMA 319(19):1999-2008. https://doi.org/10.1001/jama.2018.4 853

24. Ferrari MD, Diener HC, Ning X, Galic M, Cohen JM, Yang R, Mueller M, Ahn AH, Schwartz YC, Grozinski-Wolff M, Janka L, Ashina M (2019) Fremanezumab versus placebo for migraine prevention in patients with documented failure to up to four migraine preventive medication classes (FOCUS): a randomised, double-blind, placebo-controlled, phase 3b trial. Lancet 394(10203):1030-1040. https://doi.org/10.1016/S0140-6736(19)31946-4

25. Silberstein SD, Dodick DW, Bigal ME, Yeung PP, Goadsby PJ, Blankenbiller T, Grozinski-Wolff M, Yang R, Ma Y, Aycardi E (2017) Fremanezumab for the preventive treatment of chronic migraine. N Engl J Med 377(22):2113-2122. https://doi.org/10.1056/NEJMoa1709038
26. Goadsby PJ, Silberstein SD, Yeung PP, Cohen JM, Ning X, Yang R, Dodick DW (2020) Long-term safety, tolerability, and efficacy of fremanezumab in migraine: a randomized study. Neurology 95(18):e2487-e2499. https://doi. org/10.1212/WNL.0000000000010600

27. Rendas-Baum R, Yang M, Varon SF, Bloudek LM, DeGryse RE, Kosinski M (2014) Validation of the headache impact test (HIT-6) in patients with chronic migraine. Health Qual Life Outcomes 12(1):117. https://doi.org/10.11 86/s12955-014-0117-0

28. Lipton RB, Stewart WF, Sawyer J, Edmeads JG (2001) Clinical utility of an instrument assessing migraine disability: the migraine disability assessment (MIDAS) questionnaire. Headache 41(9):854-861. https://doi.org/10.1111/j.1 526-4610.2001.01156.x

29. Stewart WF, Lipton RB, Dowson AJ, Sawyer J (2001) Development and testing of the migraine disability assessment (MIDAS) questionnaire to assess headache-related disability. Neurology 56(Supplement 1):S20-S28. https://doi.org/10.1212/WNL.56.suppl_1.S20

30. Bagley CL, Rendas-Baum R, Maglinte GA, Yang M, Varon SF, Lee J, Kosinski M (2012) Validating migraine-specific quality of life questionnaire V2.1 in episodic and chronic migraine. Headache 52(3):409-421. https://doi.org/1 0.1111/j.1526-4610.2011.01997.x

31. Reilly MC, Zbrozek AS, Dukes EM (1993) The validity and reproducibility of a work productivity and activity impairment instrument. Pharmacoeconomics 4(5):353-365. https://doi.org/10.2165/00019053-199304050-00006

32. Coeytaux RR, Kaufman JS, Chao R, Mann JD, Devellis RF (2006) Four methods of estimating the minimal important difference score were compared to establish a clinically significant change in headache impact test. J Clin Epidemiol 59(4):374-380. https://doi.org/10.1016/j.jclinepi.2005.05. 010

33. Haan J, Hollander J, Ferrari MD (2007) Migraine in the elderly: a review. Cephalalgia 27(2):97-106. https://doi.org/10.1111/j.1468-2982.2006.01250.x

34. Burch R, Rizzoli P, Loder E (2018) The prevalence and impact of migraine and severe headache in the United States: figures and trends from government health studies. Headache 58(4):496-505. https://doi.org/1 $0.1111 /$ head. 13281

35. Lampl C, Snellman J, Ritter S, Klatt J (2020) Safety and tolerability of erenumab in older migraine patients: a subgroup analysis of randomised trials (1207). Neurology 94:1207

\section{Publisher's Note}

Springer Nature remains neutral with regard to jurisdictional claims in published maps and institutional affiliations.

Ready to submit your research? Choose BMC and benefit from:

- fast, convenient online submission

- thorough peer review by experienced researchers in your field

- rapid publication on acceptance

- support for research data, including large and complex data types

- gold Open Access which fosters wider collaboration and increased citations

- maximum visibility for your research: over $100 \mathrm{M}$ website views per year

At $\mathrm{BMC}$, research is always in progress.

Learn more biomedcentral.com/submission 